\section{Kompass \\ Dermatologie}

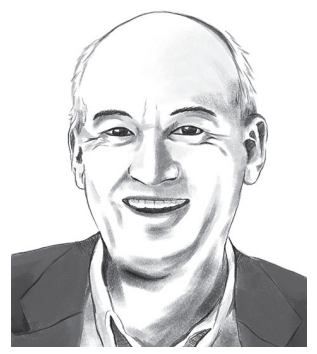

Harald Walach

Poznan/Berlin,

Polen/Deutschland

Es gibt da seit geraumer Zeit eine neue Glaubensgemeinschaft, die Pastafarier. Die Mitglieder dieser Glaubensgemeinschaft beten das fliegende Spaghettimonster an und wollen gerne als Religion akzeptiert werden, mit Spendenbescheinigung, reduziertem Steuersatz und sowas. Das machen sie natürlich nur, um Papst Franziskus und das Establishment ein wenig zu ärgern. Oder kennen Sie die Gruppierung, die sich «The Brights» nennt? Diese Bewegung hat sich laut der eigenen Webseite das Mainstreamen einer naturalistischen Weltanschauung im Namen der Wissenschaft zum Ziel gesetzt hat. Hinter beiden Vereinigungen stehen eigentlich heterogene Gruppen. Deren Kern bilden aber Leute, die im Prinzip eine neue Religion haben, nur sich dessen nicht bewusst sind. Sie sind nämlich wissenschaftsgläubig. Das hört sich dann im Originalton häufig so an: «Das wissenschaftliche Weltbild sagt uns, dass Engel, Gott, eine Seele, ein Leben vor oder nach oder hinter dem Tod, Willensfreiheit usw. nicht existieren können» oder «Wir wollen ein naturalistisch-humanistisches Menschenbild verbreiten». Was solchen Zeitgenossen meistens nicht einleuchtet: Sie verwechseln gerne die wissenschaftliche Methode (ich nenne das mal «Wissenschaft 1»)

\title{
Das Spaghettimonster und die Wissenschaft
}

mit dem Bestand dessen, was derzeit mit dieser Methode herausgefunden worden ist («Wissenschaft 2a»). Und Sie extrapolieren es dann auf das, was man vermeintlich noch oder nicht mehr entdecken kann oder wird («Wissenschaft 2b»). Dass es vielleicht einen Bestand an Erkenntnissen geben wird, der dem, was wir heute wissen und für ein wissenschaftliches Weltbild halten ( WWissenschaft 2a»), zumindest teilweise widersprechen wird oder es wenigstens ergänzen wird («Wissenschaft 3») und damit Wissenschaft 2b in die Abstellkammer der Geschichte verweisen wird, ignorieren sie gern.

\section{Als William Harvey postulierte und experimentell demonstrierte, dass das Herz eine Pumpe ist, ging ein Aufschrei durch Europa.}

Ein Beispiel: Als William Harvey postulierte und experimentell demonstrierte, dass das Herz eine Pumpe ist, ging ein Aufschrei durch Europa. Der Mainstream in Gestalt von Emilio Parisano sagte: Keiner ist in Venedig, der ein Herz schlagen hört [1]. Das finden wir heute albern, war aber so. Genauso können wir uns auch heute manches nicht vorstellen und sagen: Das ist unmöglich, undenkbar oder gar unwissenschaftlich. Aber vielleicht ist das Herz ja weder ein Konvektionserwärmer (Aristoteles), noch nur eine Pumpe (Harvey), sondern ein Verwirbler, Immunorgan und sonst noch was, was wir nicht wissen? Vielleicht ist das Gehirn ja gar kein Bewusstseinsproduktionsorgan, wie alle glauben, sondern ein Bewusstseinsmodulationsorgan? Hier kommen wir jetzt wieder zurück auf die Pastafarier. Wenn wir das, was wir mithilfe heute bestehender Methoden wissen und wissen können, verabsolutiert, wird Wissenschaft zu einer Religion und degeneriert. Dann ist Wissenschaft nicht viel mehr als der Glaube ans Spaghettimonster. Dann wird es nichts mit einer Wissenschaft 3, also mit wirklich neuen Einsichten und Erkenntnissen. Das sollten sich alle Pastafarier und die, die es noch werden wollen, zu Gemüte führen.

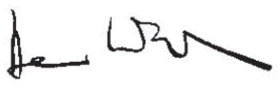

Prof. Dr. Dr. phil Harald Walach

\section{Literatur}

1 Parisano E: Recentiorum disceptationes de motu cordis, sanguinis et chyli. Leiden, Ioannis Maire, 1647, p. 107. information@karger.com

(c) 2020 S. Karger GmbH, Freiburg

www.karger.com/kkd

\section{Karger'}

Prof. Dr. Dr. phil. Harald Walach

CHS Institut

Schönwalder Straße 17, 13347 Berlin, Deutschland

hw@chs-institute.org 